\title{
Generalização da Modelagem Fracionária
}

\author{
Lucas Kenjy Bazaglia Kuroda ${ }^{1}$ \\ UNESP - Universidade Estadual Paulista, Instituto de Biociências, Botucatu, SP \\ Rubens de Figueiredo Camargo ${ }^{2}$ \\ UNESP - Universidade Estadual Paulista, Faculdade de Ciência, Bauru, SP
}

\begin{abstract}
Resumo. Este trabalho apresenta a generalização da modelagem fracionária segundo Caputo quando inserido o parâmetro de correção dimensional $(\tau)$ no modelo. Nota-se que alguns trabalhos já utilizam da nova modelagem fracionária em que o valor deste parâmetro esteja implicito. É possível assim, descrever, utilizando diferentes modelagens, curvas de soluções que estejam em maior conformidade ao dados reais.
\end{abstract}

Palavras-chave. Generalização da Modelagem Fracionária, Correção Dimensional, HPV 16, Câncer, Modelo de Gompertz.

\section{Introdução}

Na literatura, há várias definições para as derivadas fracionárias, como por exemplo, as representações de Riemann-Liouville, Riesz, Weyl, Grünwald-Letnikov, Caputo, dentre outras [6]. Uma análise minuciosa dos sistemas dinâmicos fracionários é necessária para alcançar uma definição apropriada da derivada fracionária. Por exemplo, a definição de Riemann-Liouville envolve condições iniciais fisicamente inaceitáveis (condições iniciais de ordem fracionária) [2]; ao contrário da representação de Caputo, as condições iniciais são expressas em termos de derivadas de ordem inteira com significado físico [3]. Esta definição é usada, principalmente, para incluir efeitos de memória [6]. Diante de tantos cuidados referentes a utilização do cálculo fracionário, neste trabalho será realizado a modelagem fracionária em modelos cujas soluções foram exibidas sem considerar a correta dimensão da equação quando há a troca da ordem da derivada inteira pela não inteira, baseado em [7].

\section{Generalização da Modelagem Fracionária}

A modelagem fracionária realizada em diversos trabalhos consiste em substituir a ordem da derivada inteira pela derivada fracionária, sem considerar a questão dimensional dos modelos, como por exemplo, os trabalhos de $[1,4,9,11,15,18]$. Apesar da imprecisão dimensional, os trabalhos mencionados são válidos e trazem significativas contribuições. No entanto, pode-se notar que o operador diferencial, $\frac{d}{d t}$ tem dimensão ${ }^{3} t^{-1}$ e quando realizada a modelagem fracionária $\frac{d}{d t} \rightarrow \frac{d^{\beta}}{d t^{\beta}}$

\footnotetext{
${ }^{1}$ lucaskuroda@hotmail.com

2rubens.camargo@unesp.br

${ }^{3} t$ simboliza o tempo referente a unidade de medida utilizada em modelos, podendo ser segundos, dias, horas, dentre outros.
} 
sua dimensão passa a ser $\left[\frac{d^{\beta}}{d t^{\beta}}\right]=t^{-\beta}$, o que pode tornar imprecisa a descrição do modelo, caso as constantes envolvidas já tenham sua unidade de medida fixa.

Assim, [5] propõem um procedimento alternativo simples para a construção da equação diferencial fracionária, introduzindo um novo parâmetro $\tau$ com $0<\beta \leq 1$ da seguinte maneira,

$$
\frac{d}{d t} \rightarrow \frac{1}{\tau^{1-\beta}} \frac{d^{\beta}}{d t^{\beta}}
$$

Desta forma, a equação (1) é dimensionalmente consistente, se e somente se, o novo parâmetro $\tau$, tem dimensão de tempo $[\tau]=t$. Assim, $\left[\frac{1}{\tau^{1-\beta}} \frac{d^{\beta}}{d t^{\beta}}\right]$ passa a ser uma derivada no tempo no sentido usual, cuja dimensão é $t^{-1}$.

\subsubsection{Modelos Malthusiano}

A forma mais simples de representar o processo de dinâmica populacional é pelo modelo proposto por Thomas Robert Malthus [16],

$$
\frac{d N(t)}{d t}=r N(t)
$$

$\operatorname{com} r>0$

A solução do modelo (2), é obtida via separação de variáveis, dada por

$$
N(t)=e^{r t} N(0) \text {. }
$$

A partir do modelo de crescimento tumoral de Malthus na equação (2), iremos utilizar a derivada fracionária segundo Caputo nesta equação, sendo $0<\beta \leq 1$ a ordem da derivada (consequentemente $n=1$ ) e $\tau$ o parâmetro de correção referente a dimensão da equação. Assim,

$$
\frac{1}{\tau^{1-\beta}} \frac{d^{\beta} N(t)}{d t^{\beta}}=\frac{1}{\tau^{1-\beta}}\left[{ }_{C} D^{\beta} N(t)\right]=r N(t)
$$

Sendo $F(s)$ a transformada de Laplace de $N(t)$, temos

$$
\begin{aligned}
\mathscr{L}\left[\frac{1}{\tau^{1-\beta}}\left[{ }_{C} D^{\beta} N(t)\right]\right] & =\mathscr{L}[r N(t)] \\
\frac{1}{\tau^{1-\beta}}\left[s^{\beta} F(s)-s^{\beta-1} N(0)\right] & =r F(s) \\
F(s) & =N(0) \frac{s^{\beta-1}}{s^{\beta}-r \tau^{1-\beta}} .
\end{aligned}
$$

Utilizando a transformada de Laplace inversa, temos

$$
\begin{aligned}
N(t) & =\mathscr{L}^{-1}\left[N(0) \frac{s^{\beta-1}}{s^{\beta}-r \tau^{1-\beta}}\right] \\
& =N(0) E_{\beta}\left(r \tau^{1-\beta} t^{\beta}\right) .
\end{aligned}
$$


Note que a solução analítica do modelo de Malthus (2) depende de dois parâmetros, $\beta$ e $\tau$. Assim [5,6], propõem a seguinte substituição $\tau=\frac{\beta}{r}$, com o intuito da solução analítica do modelo apresentar apenas um parâmetro, a ordem da derivada fracionária $(\beta)$. Como $0<\beta \leq 1$, temos que $0<\tau \leq \frac{1}{r}$. Daí,

$$
N(t)=N(0) E_{\beta}\left(\beta^{1-\beta}(r t)^{\beta}\right) .
$$

Assim, uma solução fracionária alternativa, com dimensão corrigida do modelo de Malthus (4) dependendo apenas do valor da derivada $\beta$ é

$$
N(t)=N(0) E_{\beta}\left(\beta^{1-\beta}(r t)^{\beta}\right) .
$$

Quando $\beta=1$, recuperamos a solução de ordem inteira do modelo, ou seja,

$$
N(t)=N(0) E_{\beta}\left(\beta^{1-\beta}(r t)^{\beta}\right)=N(0) e^{r t} .
$$

Note pela Figura 1, quanto menor a ordem de derivada, menor será o crescimento e que nas proximidades do instante inicial, todas as curvas de $\beta<1$ tem crescimento mais elevado.
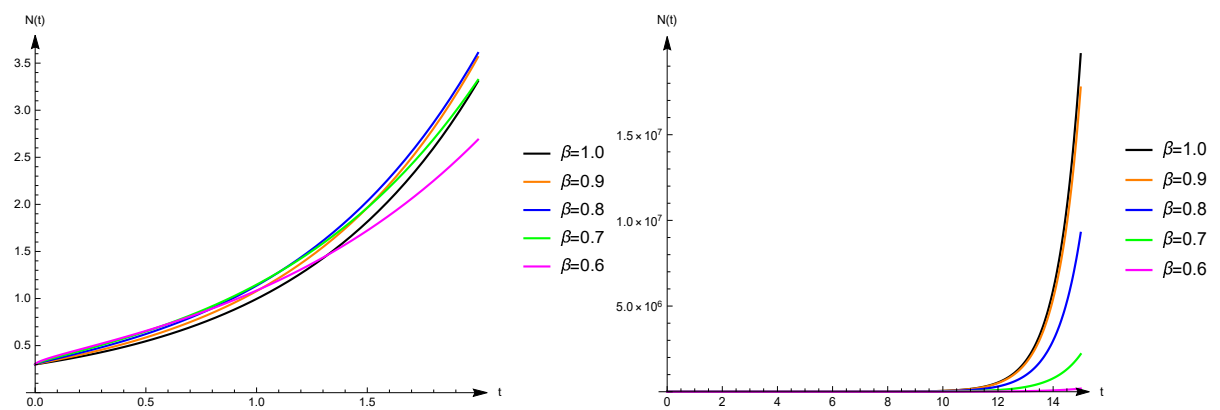

Figura 1: Solução da equação fracionária de Malthus (5) para $0 \leq t \leq 2$ e $0 \leq t \leq 15$, respectivamente.

Podemos escrever a equação de Malthus (4) de tal maneira que o parâmetro de correção dimensional esteja incluído implicitamente nas constantes do modelo, ou seja,

$$
\frac{1}{\tau^{1-\beta}}\left[{ }_{C} D^{\beta} N(t)\right]=r N(t) \Leftrightarrow{ }_{C} D^{\beta} N(t)=\beta^{1-\beta} r^{\beta} N(t) .
$$

Vejamos,

$$
\begin{aligned}
\mathscr{L}\left[{ }_{C} D^{\beta} N(t)\right] & =\mathscr{L}\left[\beta^{1-\beta} r^{\beta} N(t)\right] \\
s^{\beta} F(s)-s^{\beta-1} N(0) & =\beta^{1-\beta} r^{\beta} F(s) \\
F(s) & =\frac{s^{\beta-1} N(0)}{s^{\beta}-\beta^{1-\beta} r^{\beta}} \\
\mathscr{L}^{-1}[F(s)] & =N(0) \mathscr{L}^{-1}\left[\frac{s^{\beta-1}}{s^{\beta}-\beta^{1-\beta} r^{\beta}}\right] \\
N(t) & =N(0) E_{\beta}\left(\beta^{1-\beta}(r t)^{\beta}\right) .
\end{aligned}
$$




\subsubsection{A Escolha do Parâmetro " $\tau$ "}

$\mathrm{Na}$ seção anterior na modelagem fracionária de Caputo, foi sugerido um valor de $\tau$, em função de $\beta$, a partir do momento em que encontramos a solução da equação via transformada de Laplace. Note que em alguns casos, o desafio passa ser em encontrar o valor do parâmetro $\tau$. No entanto, esse novo parâmetro na modelagem fracionária nos abre novos horizontes sobre diferentes modelagens sem erros de dimensão se tratando do mesmo modelo. Por exemplo, referente ao modelo malthusiano (4),

$$
\frac{1}{\tau^{1-\beta}}\left[{ }_{C} D^{\beta} N(t)\right]=r N(t)
$$

quando tomado $0<\tau \leq \frac{1}{r}$ podemos reescrever o modelo da seguinte maneira, como já foi visto:

$$
{ }_{C} D^{\beta} N(t)=\beta^{1-\beta} r^{\beta} N(t)
$$

Note que a nova modelagem vai depender apenas do parâmetro $\beta$ uma vez que tomamos $\beta=r \tau$.

Agora, seja $\tau=\frac{1}{r}$ (como a dimensão de $[\tau]=\mathrm{t}$, assume-se que $\tau=\frac{1}{r}$, uma vez que $[\mathrm{r}]$ tem dimensão $t^{-1}$ ) no modelo (7), podemos reescrever esta equação da seguinte maneira,

$$
\begin{aligned}
\frac{1}{\tau^{1-\beta}}\left[{ }_{C} D^{\beta} N(t)\right] & =r N(t) \\
{ }_{C} D^{\beta} N(t) & =\tau^{1-\beta} r N(t) \\
{ }_{C} D^{\beta} N(t) & =\left(\frac{1}{r}\right)^{1-\beta} r N(t) \\
{ }_{C} D^{\beta} N(t) & =r^{\beta} N(t) .
\end{aligned}
$$

A Tabela 1 contém os modelos de Malthus (M), oscilador harmônico (OH), logístico (L), Gompertz $(\mathrm{G})$ e o modelo de von Bertalanffy (V) juntamente com algumas sugestões para o valor de $\tau$ em cada equação e sua respectiva modelagem para o valor do parâmetro escolhido $(\tau)$. Encontra-se também alguns trabalhos em que o valor do parâmetro foi utilizado, mesmo que implicitamente ${ }^{4}$.

Nota-se que, na nova modelagem fracionária apesar de surgir mais um parâmetro no modelo $(\tau)$, podemos descrever diversas soluções e aproximar essas curvas ao dados reais, como será visto a seguir. Para modelos mais complexos se faz necessário a utilização de métodos computacionais para encontrar a solução fracionária, como por exemplo, o método de Grünwald-Letnikov [14] utilizado neste trabalho.

\subsubsection{Aplicação - Modelo referente ao HPV 16}

O modelo matemático (8) é referente à infecção por papilomavírus humano (HPV 16) administrados em camundongos [10]. Utilizaremos três tipos de modelagem fracionária de Caputo e exibiremos a melhor aproximação aos dados reais.

$$
\frac{d T(t)}{d t}=\frac{1}{\mu_{g}} \ln \left[\frac{\ln \left(\theta_{g} / T_{0}\right)}{\ln \left(\theta_{g} / 2 T_{0}\right)}\right] T(t) \ln \left(\frac{\theta_{g}}{T(t)}\right),
$$

sendo $T(t)$ o volume do tumor $\left(\mathrm{mm}^{3}\right)$ no tempo t, $\theta_{g}$ o tamanho máximo tumoral $\left(\mathrm{mm}^{3}\right), \mu_{g} \mathrm{o}$ tempo de duplicação do tumor (dias) e $T_{0}$ o tamanho inicial do tumor.

\footnotetext{
${ }^{4}$ Modelagem utilizada sem citar o valor do parâmetro $\tau$.
} 
Tabela 1: Modelagem para diferentes valores de $\tau$.

\begin{tabular}{|c|c|c|c|c|}
\hline Modelo & Equação & $\tau$ & Modelagem & Referência \\
\hline M & $\frac{1}{\tau^{1-\beta}}\left[C^{D^{\beta} N(t)}\right]=r N(t)$ & $\tau=\frac{1}{r}$ & $C^{D^{\beta} N(t)}=r^{\beta} N(t)$ & {$[8,19]$} \\
\hline $\mathrm{OH}$ & $\frac{1}{\tau^{2-\beta}}\left[C^{D^{\beta} x(t)}\right]=-\omega^{2} x(t)$ & $\tau=\frac{\beta}{\omega}$ & $C^{D^{2 \beta} x(t)=-\beta^{2(1-\beta)} \omega^{2 \beta} x(t)}$ & [5] \\
\hline $\mathrm{OH}$ & $\frac{1}{\tau^{2-\beta}}\left[C^{D^{\beta} x(t)}\right]=-\omega^{2} x(t)$ & $\tau=\frac{1}{\omega}$ & $C^{D^{\beta} x(t)}=-\omega^{\beta} x(t)$ & [13] \\
\hline L & $\frac{1}{\tau^{1-\beta}}\left[C^{D^{\beta} N(t)}\right]=r N(t)[1-N(t)]$ & $\tau=\frac{1}{r}$ & $C^{D^{\beta} N(t)}=r^{\beta} N(t)[1-N(t)]$ & [12] \\
\hline G & $\frac{1}{\tau^{1-\beta}}\left[C D^{\beta} N(t)\right]=r N(t) \ln \left(\frac{k}{N(t)}\right)$ & $\tau=\frac{1}{r}$ & $C^{D^{\beta} N(t)}=r^{\beta} N(t) \ln \left(\frac{k}{N(t)}\right)$ & - \\
\hline $\mathrm{V}$ & $\frac{1}{\tau^{1-\beta}}\left[C^{D^{\beta}} V(t)\right]=a V(t)^{\frac{2}{3}}-b V(t)$ & $\tau=\frac{3 \beta}{b}$ & - & {$[17]$} \\
\hline
\end{tabular}

\section{Modelagem Fracionária 1 (MF1)}

Nesta modelagem, $\tau$ e $\beta$ surgem como dois novos parâmetros do modelo:

$$
\frac{1}{\tau^{1-\beta}} \frac{d^{\beta} T(t)}{d t^{\beta}}=\frac{1}{\mu_{g}} \ln \left[\frac{\ln \left(\theta_{g} / T_{0}\right)}{\ln \left(\theta_{g} / 2 T_{0}\right)}\right] T(t) \ln \left(\frac{\theta_{g}}{T(t)}\right) .
$$

\section{Modelagem Fracionária 2 (MF2)}

Assumiremos que $\tau=\frac{1}{r}$, sendo $r=\frac{1}{\mu_{g}} \ln \left[\frac{\ln \left(\theta_{g} / T_{0}\right)}{\ln \left(\theta_{g} / 2 T_{0}\right)}\right]$. Assim, a MF2 será, como visto na Tabela 1 ,

$$
\frac{d^{\beta} T(t)}{d t^{\beta}}=r^{\beta} T(t) \ln \left(\frac{\theta_{g}}{T(t)}\right) \text {. }
$$

\section{Modelagem Fracionária 3 (MF3)}

Como visto na Tabela 1 assumindo $\tau=\frac{\beta}{r}$, a MF3 tem a forma,

$$
\frac{d^{\beta} T(t)}{d t^{\beta}}=\beta^{1-\beta} r^{\beta} T(t) \ln \left(\frac{\theta_{g}}{T(t)}\right),
$$

sendo $r=\frac{1}{\mu_{g}} \ln \left[\frac{\ln \left(\theta_{g} / T_{0}\right)}{\ln \left(\theta_{g} / 2 T_{0}\right)}\right]$.

Na Figura 2 está a simulação da modelagem fracionária 1 e 3. Os parâmetros utilizados foram a partir da estimuação dos parâmetros do HPV 16 de [10]. Assumi-se para o primeiro tumor $T_{0}=5,69, \theta_{g}=106 \mathrm{~mm}^{3}$ e $\mu_{g}=27,251$ e para o segundo tumor $T_{0}=4,36, \theta_{g}=106 \mathrm{~mm}^{3} \mathrm{e}$ $\mu_{g}=19,439$. Obs: A modelagem 2 não foi exibida no gráfico por estar bem próxima da modelagem 3 .

Nota-se que nas modelagens 1, 2 e 3, foi possível ajustar a curva de solução mais próximas aos dados reais na descrição do volume tumoral pela infecção do HPV 16. 

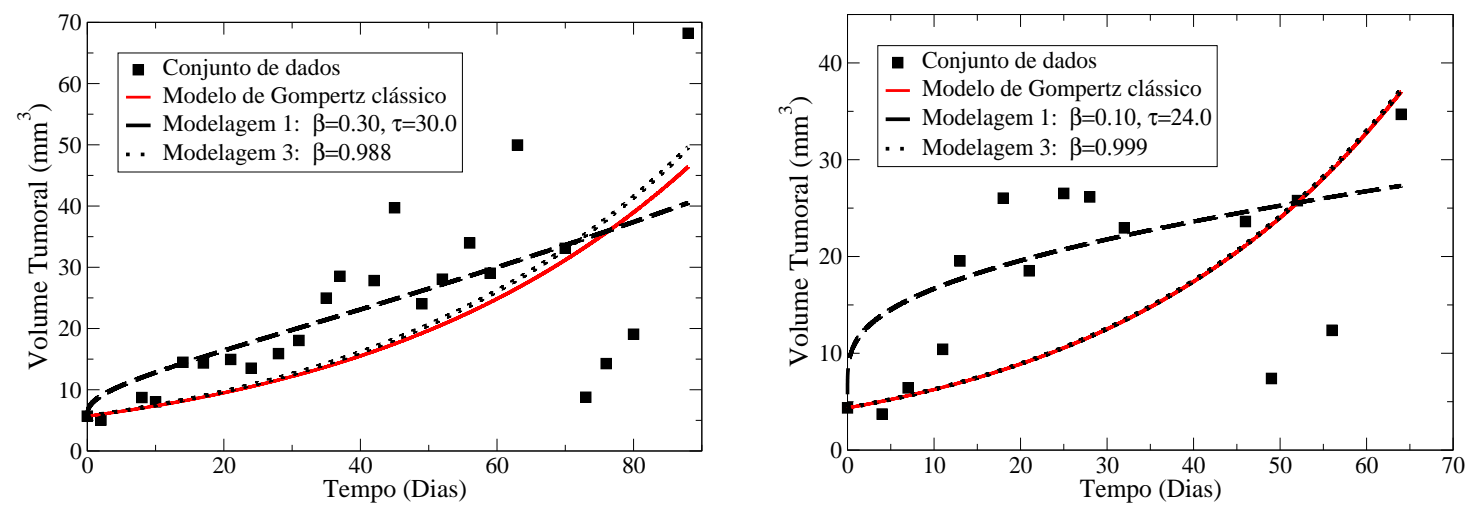

Figura 2: Curvas de crescimento tumoral.

\section{Conclusões}

Neste trabalho foram utilizados três casos de modelagem fracionária, que surgiram a partir da generalização da modelagem fracionária quando inserimos o parâmetro $\tau$ de correção dimensional. Nota-se que é possivel descrever diversas solução e buscar a que mais se aproxima ao dados reais. A primeira modelagem foi a mais adequada no ajuste aos dados reais, já que sua raiz do erro quadrático médio (RMSE) é igual a $R M S E=11,2085$, a modelagem 2 e 3 possuiram aproximadamente $R M S E=12,5655$. Já no modelo usal $R M S E=12,6236$. Portanto, em todas as modelagens aqui descritas, foi possível ajustar a curva de solução mais próximas aos dados reais na descrição do volume tumoral pela infecção do HPV 16.

\section{Agradecimentos}

O presente trabalho foi realizado com apoio da Coordenação de Aperfeiçoamento de Pessoal de Nível Superior - Brasil (CAPES) - Código de Financiamento 001, Processo: 33004064083P2. Agradecemos também o grupo de pesquisa CF@FC e ao Instituto Federal, Campus São Roque.

\section{Referências}

[1] Camargo, R. F. and Oliveira, E. C. Cálculo fracionário. Editora Livraria da Física, São Paulo, 2015 .

[2] Diethelm, K. The analysis of fractional differential equations: An application-oriented exposition using differential operators of Caputo type. Springer, Berlin, 2010.

[3] Dielthelm, K. and Ford, N. J. and Freed, A. D. and Luchko, Y. Algorithms for the fractional calculus: A selection of numerical methods, Computer Methods in Applied Mechanics and Engineering, v.194, p.743-773, 2005.

[4] El-Sayed, A. M. A. and El-Mesiry, A. E. M. and El-Saka, H. A. A. One the fractional-order logistic equation, Applied Mathematics Letters, v.20, p.817-823, 2007. 
[5] Gómez-Aguilar, J. F. and Rosales-García, J. J. and Bernal-Alvarado, J. J. and Córdova-Fraga, T. and Guzman-Cabrera. Fractional mechanical oscillators, Revista Mexicana de Fúsica, v.58, n.9, p.348-352, 2012.

[6] Gómez-Aguilar, J. F. and Yepez-Martínez, H. and Ramón, C. C. and Orduna, I. C. and Jiménez, R. F. E. and Peregrino, R. F. E. Modeling of a mass-springdamper system by fractional derivatives with and without a singular kernel, Entropy, v.17, n.9, p.6289-6303, 2015.

[7] Kuroda, L. K. B. Nova modelagem fracionária aplicada à dinâmica tumoral (HPV 16), Tese de doutorado, UNESP - Botucatu, 2020.

[8] Kuroda, L. K. B. and Bruno-Alfonso, A. and Mancera, P. F. A. and Camargo, R. F. Análise do método multi-passos com transformada diferencial generalizada na modelagem fracionária, TEMA - Trends in Applied and Computational Mathematics, v.20, n.1, p.133-147, 2019.

[9] Kuroda, L. K. B. and Gomes, A. V. and Tavoni, R. and Macera, P. F. A. and Varalta, N. and Camargo, R. F. Unexpected behavior of Caputo fractional derivative, Computational and Applied Mathematics, v.36, n.3, p.1173-1183, 2017, doi:10.1007/s40314-015-0301-9.

[10] Loizides, C. and Iacovides D. and Hadjiandreou, M. M. and Rizki, G. and Achilleos, A. and Strati, K. and Mitsis, G. D. Model-based tumor growth dynamics and therapy response in a mouse model of De Novo carcinogenesis, PloS One, v.10, n.12, p.81-95, 2015.

[11] Mainard, F. Fractional relaxation-oscillation and fractional diffusion-wave phenomena, Chaos, Solitons and Fractals, v.7, n.9, p.1461-1477, 1996.

[12] Ortigueira, M. and Bergochea, G. A.A new look at the fractionalization of the logistic equation, Physica A, v.467, p.554-561, 2017.

[13] Rodrigues, F. G. and Oliveira, E. C. Introdução às técnicas do cálculo fracionário para estudar modelos da física matemática, Revista Brasileira de Ensino de Física, v.37, n.3, p.1-12, 2015.

[14] Scherer, R. and Kalia, S. L. and Tang, Y. and Huang, J. The Grünwald-Letnikov method for fractional differential equations, Computers and Mathematics with Applications, v.62, p.902-917, 2011.

[15] Sólis-Péres, J. E.and Gómez-Aguilar, J. F. and Escobar-Jiménez, R. F. and Olivares-Peregrino, V. H. Parameter estimation of fractional gompertz model using Cuckoo search algorithm. Fractional derivatives with Mittag-Leffler kernel, Fractional Derivatives with Mittag-Leffler Kernel, v.194, p.81-95, 2019.

[16] Seti, B. D. and Betencourt, M. F. B. and Oro, N. T. and Krioka, R. M. L. and Muhl, V. J. L. Estudo da dinâmica populacional usando os modelos de Malthus e Verhulst: Uma aplicação à população de Passo Fundo, Revista Teoria E Evidência Econômica, v.7, n.12, p.137-143, 1999.

[17] Tavoni, R. Modelos fracionários de terapia gênica para o tratamento do câncer, Tese de doutorado, UNESP - Botucatu, 2019.

[18] Veeresha, P. and Prakasha, D. G. and Baskonus, H. M. New numerical surfaces to the mathematical model of cancer chemotherapy effect in Caputo fractional derivatives, Chaos: An Interdisciplinary Journal of Nonlinear Science, v.29, n.1, p.1-15, 2019.

[19] West, B. J. Exact solution to fractional logistic equation, Physica A, v.429, p.103-108, 2015. 